\title{
IV. Ueber die Wirkung nicht-leitender Körper bei der elektrischen Induction ${ }^{1}$ ); con M. Faraday und P. Riefs.
}

(Philosoph. Magas. Ser. IV. Fol. XI. p. 1.)

Der nachfolgende Brief erklärt sich selbst. Ich habe darauf eine sehr verbindliche Antwort von Hrn. Riefs erhalten, welche ich demselben hinzuzufügen wünsche. Ich habe das Englische der Antwort ein wenig geändert, hauptsïchlich in einzelnen kleinen VVorten und nur in solchen Fällen, wo ich glaubte, die Aenderung würde die Meinung des Verfassers klarer machen. Gewisse Ausdrücke von Hrn. Riefs verlangen fast eine Antwort. In Bezug auf diese Fälle und um Zweidentigkeit in Betreff meiner eignen Meinung zu entfernen, habe ich gewagt, einige Anmerkungen hinzuzufügen, nicht um eine Discussion über Hypothesen zu erregen, sondern um meine Ansicht (dem Leser) zu erlïutern. Es ist nicht die Pflicht oder das Amt eines Naturforschers Glauben zu dictiren, und jede Hypothese ist mehr oder weniger Sache des Glaubens; er hat nur seine Thatsachen und seine Schlüsse zu geben, und so viel von der Logik, welche die crsteren mit den letzteren verknüpft, als er für nothwendig halten mag; dann hat er das Ganze der wissenschaftichen Velt zur gegenwärtigen, und, wie er zuweilen ohne Anmafsung glauben darf, zur künftigen Beurtheilung zu überliefern

London, 19. Nov. 1855.

E ben erst habe ich lhren Aufsatz über die Wirkung nichtleitender Körper bei der elektrischen Induction kennen gelernt; ich hatte ihn zwar schon in Poggendorff's Annalen gesehen ${ }^{2}$ ), vermochte ihn aber nicht zu lesen. Im Juniheft des Philosophical Magazine d. J. Vol. IX. p. 401 ist jedoch eine Uebersetzung davon erschienen, und aus dieser ersehe ich, dafs es mir nicht gelungen ist, lhnen (und deshalb vermuthlich auch Anderen) meine wahre Meinung beizubringen, vielmehr das, was sie für meine Ansicht halten, in einigen sehr wichtigen Punkten absolut das Gegentheil davon ist. Sie werden sich nicht wundern, dafs es mir daran liegt, mich in einer solchen Sache zu rechtfertigen gegen Jemand, der eine so hohe Stellung in der Wissen-

1) D. h. hier immer: Elektricitätsvertheilung.

2) Es ist der Aufsatz in d. Ann. Bd. 92, S. 377.

$\boldsymbol{P}$.

$\boldsymbol{P}$. 
schaft einnimmt wie Sie. Ich mufs mich hiebei auf das Philosophical Magazine beziehen, denn wenn ich auch die Richtigkeit der Uebersetzung nicht zu beurtheilen vermag, habe ich doch keinen anderen Weg zu Ihrem Aufsatz zu gelangen.

Unten auf $p .402$ sagt der Aufsatz, Faraday habe sich bemüht, dic Ansicht durchzuführen "that induction is not produced by the action of electricity across space, but that an electric body acts only on the contiguous particles of an insulating medium, “ etc. Wenn Sie meine Aufsätze nachschlagen, so werden Sie finden, dafs ich ganz zu Anfange des über Induction (1165) ') diese Ansicht speciell auf die Fälle der gewöhnlichen Induction beschränkt habe, d. b. auf Fälle, wo. Materie zugegen ist; in (1215) ist dieser Ausdruck wiederholt, und abermals in den Experiment. Research. Vol. II, p. 267. Statt zu sagen, Induction könne nicht querdurch den Raum gescheben, habe ich speciell von dem Fall eines Vacuums gesprochen $(1613-1616)$, welcher Fall in einem Briefe an Dr. Hare (Exp. Res. Vol. II, p. 262, 266) weitläufiger betrachtet ist.

Pag. 403 des Phil. Mag. sagt Ihr Aufsatz: "It follows from other experiments made by Faraday (1218) that the induction would have been diminished had a conducting plate been introduced between the two; for, according to Faraday's opinion, the introduction of a conducting plate would have caused the induction to take place in curved lines around the edges of the plate, instead of in right lines through the intervening stratum of air." Wenn diese Uebersetzung Ihre Meinung ausspricht, so kann ich nicht finden, welcher Ausdruck von mir Sie verleitet hat zu glauben, das Obige sey meine Meinung. Nirgendwo habe ich gesagt oder angedeutet, dafs die Einschiebung ciner solchen Platte die Induction vermindere oder sie veranlasse nur in um die Ränder derselben herumbiegenden

1) Die hier citirten Paragraphen finden sich in des Hrn. Verf. 11 ter, 12ter und 13ter Reihe von Untersuchungen, die im Bd. 46, 47 und 48 dies. Ann, enthalten sind.

$\boldsymbol{P}$. 
oder mehr als zuvor gekrümmten Linien stattzufinden. Im Gegentheil weifs ich, dafs vermöge einer solchen Platte mehr Kraftlinien durch den von ihr eingenommenen Raum gehen als zuvor, dafs, was diesen Theil des Raumes betriff, die Induction durch die bessere Function der Leitung ersetzt seyn wird, dafs diese, statt die Induction zu hemmen, das Endresultat begünstigen wird, obgleich dieses Resultat durch die Gröfse und Gestalt der Platte, durch die Abstände von ibr und den wirkenden Körpern und durch andere Umstände verwickelt werden würde, wie es Ihr Aufsatz so gut nachweist.

Der Fall von mir, auf welchen Ihr Aufsatz sich oben (1218) bezieht, ist einer von denen, welchen ich suchte, als die Möglichkeit der Induction in krummen Linien darthuend, und er ist nicht angeführt als Beweiz, dafs sie stets in krummen Linien gescheben müsse, was meinen Gedanken sehr fern liegt. Bei ihm ist das erwähnte Metallstïck (Kugel, Halbkugel oder Platte) nicht isolirt, sondern abgeleitet (uninsulated) (1218-1230). Der Leiter bildet auch das Ende der Induction und ist nicht in die Bahn der Induction eingeschoben; Fälle so verschiedener Art, dafs viel des Räsonnements, welches für den einen gilt, keine Beziehung zu dem anderen hat. Der letztere Fall ist, weil ich ihn für allgemein bekannt hielt, nicht speciell in den Experiment. Research. angeführt, wohl aber in dem Briefe an Dr. Hare (Collect. Exp. Res. Vol. II. p. 163 ).

Vielleicht wird Ihnen die folgende Auffassungsweise meine Ansichten klar machen. Sey $P$ ein isolirter gelade-

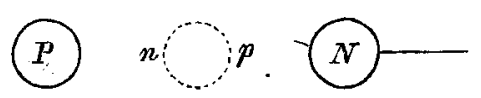
ner Körper, der auf $N$, einen unisolirten metallischen Körper, inducirend wirkt; $n p$ sey anfangs nicht da. Nun werde $n p$ eingeschoben; es sey ein Nichtleiter wie Schellack oder Schwefel, aber von derselben specifischen inductiven Capacität wie Luft. Es wird in der Anordnung der Kräfte keine Verănderung eintreten, denu die Theilchen von $n p$ werden genau so polarisirt, wie es

Poggendorff's Annal. Bd. XCVII. 
die durch sie verdrängten Lufttheilchen waren. Hierauf denke man sich $n p$ mit Leitungsvermögen versehen, wie. wenn es in ein Metall verwandelt worden wäre. Seine Theilchen werden sich nun gegen einander entladen; die Theile bei $n$ und $p$ werden negativer und positiver seyn, als sie zuvor waren, weil der Inductions-Abstand zwischen $P$ und $N$-um den Durchmesser von $n p$ verkürzt ist; so wird die Induction stärker seyn, und statt dafs die Kraftlinien von $P$ nach $N$ um $n p$ herumgehen (wie $\mathrm{lhr}$ Aufsatz mich sagen lä(st), werden jetzt, da der Raum $n p$ ein Leiter ist, mehr von ihnen auf denselben kommen und durch ihn gehen als zuvor, da er'ein Isolator war (1326. 1337. 1338). Sicher brauche ich hiebei nicht länger zu verweilen, denn ich bin überzeugt, dafs Sie, wenn Sie die Paragraphen 1218 - 1230 oder vielleicht auch Exp. Res. Vol. IJ. p. 279 - 84 nachlesen, sogleich sehen werden, was meine Meinung war und ist. Nach ihr sind die Resultate genau so, wie Sie dieselben im Phil. Mag. pp. 406 und 407 beschreiben.

In Ihrem Aufsatz (Phil. Mag. p. 410) beschreiben Sie einen Versuch, welchen ich wohl kenne und als einen der stärksten Beweise der Richtigkeit meiner Ansichten be trachte. Eine Schellackplatte wird mit ibrer Vorderseite 12 Zoll von dem positiven Koopf einer Elektrisirmaschine, und wit ihrer Hinterseite 1 Zoll von der Flamme einer Weingeistlampe entfernt aufgestellt, und darauf, vor ihr herumgelübrt. Nach Fortnahne und Untersuchung finden Sie die Vorderseite negativ geladen, und daraus schliefsen Sie, dafs, vor der Entladung der Minterfläche durch die Flamme, die Induction die Vorderseite des Schellacks negativ, und die Hinterseite positiv gemacht habe, genau wie es bei einer Metallplatte geschehen seyn würde, und, soweit ich Ihren Aufsatz verstebe, vermöge eines gleichen Leitungsactes durch seine Masse hin. Meine Ansicht von der Induction kommt nun in Bezug auf die Vorder-und Hinterfläche des Schellacks mit der Ibrigen überein; aber in zwei wichtigen Punkten weicht sie von ihr ab. Sie 
nimmt an, dafs, wenn vorausgesetzt wird, die Platte bestebe aus einer Uuzahl von parallelen Platten und jede dieser aus einer einzelnen Lage von Theilchen, jede Platte ihre vordere negative und ihre hintere positive Fläche habe; und dafs dic Positivität der äufseren Hinterfläche nicht aus der Fortpflanzung der Flektricität durch die zwischen ihr und der negativen Vorderfläche liegenden leitenden Theilchen entspringt, sondern aus der Fortpflanzung der Kraft durch die Polarität der isolirénden Theilchen. So anfgefafst, ergiebt sich aus diesem Fall ein Paar. Betrachtungen. geeignet zur Prüfung des relativen Werthes der beiden Ansichten, und bisjetzt bestärken sie mich in der meinigen.

Hätte die Schellackplatte ihre Vorderfläche geladen, wie eine metallene oder leitende Platte die ibrige geladen haben würde, so würde jene Fläche, nach Fortnahme der Platte aus der Induction, nicht ausschliefslich geladen geblieben seyn; vielmehr wäre die Schellackplatte, gleich einer leitenden Platte, auf beiden Seiten und ihrer ganzen Oberfläche, geladen gefunden worden; denn dieselbe Leitung, welche während der Induction den Fluf́s der Elektricität gestattete, würde auch nach der Induction die Rückkehr derselben zu allen Punkteu erlauben. Da nicht für den einen Theil des Versuchs Leitung angenommen, und für den anderen abgelehnt werden kann, so halte ich schon diese Betrachtung für tödtlich für Ihre Ansicht, so wie ich sie aus der Uebersetzung verstehe.

Die zweite Betrachtung ist folgende. Wenn die Schellackplatte, während sie in der Inductionslage befindlich ist, nach meiner Ansicht als eine Masse nicht-leitender polarisirter Theilchen betrachtet werden kann, so wird die Wirkung der Weingeistflamıe negative Elektricität durch Fortführung (convection) zur Hinterfläche der Platte zu schaffen haben, um während der Zeit ihren temporären gezwungenen positiven Inductionszustand zu neutralisiren; und es ist diese Fläche, welche (nach der Fortnahme der Platte aus der Induction, und der Rückkehr aus dem nicht länger von $P$ unterhaltenen gezwungenen Zustande) als negativ geladen be- 
trachtet werden mufs, nicht die Vorderfläche, indem nun die letztere nur durch die noch übrig gebliebene Polarität der Theilchen zwischen ibr und der wirklich geladenen Hinterfläche im relativ negativen Zustand gehalten wird. So können, für sich und aufser dem von der Leitung entnommenen Argument, andere bedingende Betrachtungen erhoben werden. Wenn Ihre Ansicht die richtige ist, so ist es nur die Vorderfläche, welche negativ geladen wird und $z w a r$ durch eine halb entladene inductive Action; nach meiner Ansicht ist es die Hinterfläche, welcher dieser $\mathrm{Zu}$ stand durch Fortführung (convection) von der Flamme zugeführt wird. Nach Ihrer Ansicht befinden sich die inneren Theile der Platte in ihrem natürlichen Zustande; nach der meinigen sind sie noch polarisirt und werden in diesem Zustande durch die hintere negative Ladung erhalten. Glücklicherweise kann die Frage, ob die Vorder-oder die Hinterfläche negativ geladen worden, beantwortet werden, obwohl nicht durch indifferente Annäherung einer der Seiten der Platte an ein Goldblatt-Elektrometer; denn mit jeder der Seiten werden Anzeigen von negativer Elektricität erhalten; und wenn die erregte Fläche in beiden Fällen gleiche Abstände von der Elektrometerkappe besitzt, wird die Wirkung grölser seyn (wegen specifischer inductiver Capacität), wenn der Körper der Schellackplatte zwischen der Kappe und der erregten Fläche steht, als wenn blofs Luft dazwischen ist.

Um diese Punkte ein für alle Mal klar zu machen, will ich die gebrauchten Platten beschreiben, und, zum leichten Verständnifs der Lage, eine Figur von den Formen des Experiments geben. Eine Platte war von Schellack, $4 \frac{1}{2}$ Zoll im Quadrat und 0,9 Zoll dick, die andere von Schwefel, 5 Zoll im Quadrat und 0,8 Zoll dick. Ein starker Faden weifser Seide war rund um den Rand jeder Platte gebunden und dann eine lange Schleife von derselben Seide an den beiden Ecken einer Seitenkante befestigt; diese Schleife und eine gleiche an den beiden Ecken der gegenüberliegenden Seitenkante dienten als Handhahen, durch welche 
die Platten isolirt und doch herumgeführt oder in jede Lage -gegen das Elektrometer gebracht werden konuten. In der Figur ist $S$ eine dieser Platten, längs der Kaute

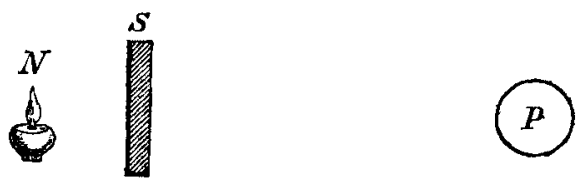

gesehen; $P$ stellt den inducirenden oder urspringlich geladenen Körper vor und $N$ den inducirten Körper (Flamme, Spitze, Hand oder Kugel). Zwischen $\boldsymbol{P}$ und $\boldsymbol{N}$ findet die Iuduction statt, welcher, soweit es die Resultate des Versuchs betrifft, die Platte $S$ unterworfen ist und deren Effecte zu untersuchen sind. Die Resultate sind bei beiden, der Schwefel- wie der Schellackplatte, genan gleich. War $P$ negativ gemacht, so waren sie auch gleich, nur von umgekehrteu Zeichen. Ich will die mit dem Schellack erhaltenen beschreiben, und dabei stets die $P$ zugewandte Seite die Vorderfläche, und die $N$ zugewandte die Hinterfläche der Platte uennen.

Als $P$ das Ende des positiven Conductors einer Elektrisirmaschine und $N$ eine unisolirte metallene Kugel oder Platte war, wurde die Schellackplatte in ihre Lage gebracht und dreifsig Sekunden oder länger darin gelassen; daun fortgenommen und mittelst eines Goldblatt-Elektrometers geprüft, erwies sie sich auf jeder Fläche oder jedem Theil vollkommen ungeladen. Darauf wurde die Schellackplatte wieder in ihre Stellang in Induction gebracht, und in $N$ eine Weingeistflanme in der von Ihuen bescbriebenen Weise angewandt. Fortgenommen, wurde die Schellackplatte untersucht, indem man sic obne Reibung auf die Deckplatte des Elektrometers legte. Der Schellack ertheilte den Goldblättern eine starke negative Ladung, welche Seite auch auf der Kappe liegen mochte; doch waren die Anzeigen bei weitem am stärksten, wenn die Hinterseite die Kappe berührte, zum Beweise, dafs, so weit sie ging, die Ladung wirklich an dieser Seite.stattgefunden hatte. 
Nach meiner Ansicht von der Induction ist diese Seite der Platte durch die Flamme negativ geladen worden. Denn der Theil der Induction zwischen $S$ und der Flamme kounte durch die von der letzteren abhängige Fortfübrung (ćonvection) zerstört werden, wenn zwischen jhr und $P$ blofs Luft vorhanden gewesen wäre. Allein da die Luft in Richtung der Induction begränzt war durch die Hinterfläche des Schellacks, so konnte die Flamme ihren Ladungszustand blofs bis zu dieser Fläche führen, und das Isolationsvermögen und die Solidität des Schellacks verhinderten. fernere Veränderungen in dieser Richtung. Daraus das schon beschriebene Resultat. Da die Flamme das Vermögen besitzl, die Hinterfläche zu laden, so kann sic auch dieselbe entladen, und daher wird, wenn man die Flamme einen Moment parallel jener Fläche und etwa einen Zoll von ihr hin - und herführt, die Platte gänzlich entladen werden. Der frübere negative Ladungszustand der Hinterfläche der Platte wird, wenn man es wünscht, eine Minute oder zwei, und selbst fünf oder zehn Minuten verweilen, und dennoch entladet sie der momentane Gebrauch der Flamme gänzlich. Das Resultat stimmt vollkommen mit meiner Ansicht, scheint mir aber der Ihrigen gänzlich zu widersprechen. Auch kann ich nicht einsehen, wie dic Annahme von irgend einem Grade der Leitung in dem Schellack, der mit den anerkannten Thatsachen seines Isolationsvermögens verträglich wäre, das Resultat zu erklären vermöchte.

Allein man könnte sagen, die zweite Anwendung der Flamme habe die negative Hinterfläche statt sie zu entladen, wirklich positiv geladen, in Betrage gleich der vorausgesetzten negativen Ladung der Vorderfläche, und überdecke somit den Effect der letzteren mehr oder weniger je nach der Dicke der Platte. Dann fragt es sich: Ist die Platte gänzlich cntladen oder ist sie nun doppelt geladen, d. h. mit der einen Fläche positiv, und mit der anderen negativ? Ich finde sie gänzlich entladen, denn wenn ich eine der Oberflächen auf die Deckplatte des Elektrometers bringe und an die andere Fläche sorgfältig eine unisolirte Metallplatte, 
linde ich keine Wirkung aul das Elektrometer, wogegen solche Wirkung da seyn würde, wenn die Platte wie eine Leydener Flasche geladen worden wäre.

Oder man köunte annehnen, die zweite Anwendung der Flamme, obwohl auf die Hinterfläche des Schellacks gerichtet, habe auf eine oder die andere Weise die negative Vorderfläche entladen. Dal's diel's nicht der Fall ist, läfst sich leicht zeigen durch Anwendung der Flamme auf die Vorderseite, und dann kommen noch stärkere Beweise als die schon erhaltenen gegen Itre und für meine Ansicht zum Vorschein; denn, nach meiner Ansicht, müfste eine solche Anbringung der Flamme die Vorderfläche veranlassen. eine positive Ladung anzunchmen, in sofern ein zweiter Inductionsfall errichtet ist, in welchem die hintere negativ geladene Fläche des Schellacks der inducirende Körper ist. gegen welche die Flamme wie zuvor den inducirten Theil spielt, und durch ihre wohlbekannten Fortführungskräfte (powers of convection) ibren Ladungszustand auf die (früher vordere), jetzt ihr zugewandte Oberfläche des Schellacks überträgt (transfers); wogegen sie nach Ihrer Ansicht einfach entladen werden miifste. Die Schellackplatte wurde deshalb vor dem geladeven Körper $P$ aufgestellt, und die Flamme vor ihrer Hinterfläche herumgefülırt; dann wurde die Platte aus der inductiven Stellung genommen, die Flanme vor ihrer Vorderfläche herungeführt, und darauf die Platte mittelst des Elektrometers untersucht. Wenn die Vorderfläche auf der Deckplatte des Instruwentes war, z.eigte dieses eine positive Ladung; berührte dagegen die Hinterfläche die Kappe, so zeigte das Instrument eine negative Ladung, also dieselbe Art von elektrischer Ladung für diese Fläche als zuvor, aber eine viel schwächere. Die scheinbare Schwäche mufste eintreten, denn nun wurde die negative Ladung der Hinterfläche inductive quer durch den Schellack gegen die positive Ladung der Vorderfläche aus. geübt, und vice versa. Und dafs diefs der Fall sey, wurde bewiesen, indem man die Hand oder eine unisolirte Metallplatte gegen die obere Vorderfläche brachte, während die 
Hinterfläche mit der Kappe des Elektrometers in Berührung war; denı dic negative Divergenz der Goldblättchen vergröfserte sich sebr, da die negative Elektricität in zu grofsem Maafse durch die Induction der positiven Vorderfläche in Freiheit gesetzt wurde. Und wenn die positive Vorderfläche mit der Kappe des Elcktrometers in Berülirung war, konnte dessen starke Ladung in gleicher Weise nachgewiesen werden. Sonach kann also die Flamme, sorgfältig genähert, nur die Seite, welche eine Ladung erhalten bat, entladen, und das nur, wenn sie dieser Seite genähert wird; bringt man sie der anderen Seite gegenüber, so führt sic ihr die entgegengesetzte Elektricität zu, und hinterläfst die Platte doppelt geladen.

Diese Versuche sind keineswegs schwierig oder zart; sie lassen sich (mit wentgen einfachen Vorsichtsınaafsregeln) auf die überzeugendste und mannigfaltigste Weise anstellen, und geben immer dasselbe Resultat. $P$ oder der inducirende Körper ist am besten von grofser Oberfläche. Ein geriebener (excited) Glasstab oder, noch besser, eine geriebene Guttapercha-Platte (eine Schuhsoble) ist sehr zweckmäfsig. Man kann damit dem Schellack oder Schwefel $S$ bis auf einen Zoll nahe kommen, ohne ihm eine Ladung mitzutheilen, sobald keine entladenden Leiter in der Nähe sind. Statt der Flamme kann man bei $N$ eine feine Metallspitze anwenden, kann auch Leiter und Contact in folgender Weise gebrauchen. Ein Goldblatt wurde auf die Deckplatte des Elektrometers gelegt, $P$ an seinen Ort gebracht, ebenso die Schellack- oder Schwefelplatte $S$, und die Flamme für einen Moment in $N$ aufgestellt. Hierauf wurde die Platte $S$ entfernt und mit ihrer negativ geladenen Hinterfäche mit dem Goldblatt auf dem Elektrometer in Berübrung gesetzt. Sogleich zeigte das letztere eine starke Divergenz (oft eine stärkere als das Instrumeut, ungeachtet es sehr grofs war, ertragen konnte); weun überdiefs ein unisolirter Draht dem Goldblatt oder der Kappe genähert wurde, entlud sich die geladene Hinterfläche mit eiuem Funken, .und Elektrometer und Schellack 
blieben vollkommen ungeladen zurück. Es ist nur ein sehr kleiner Schritt, die Hinterfläche zuvor mit Zinnfolie zu belegen, und dann lassen sich alle Versuche beim Contact mit einem unisolirten Körper, statt der Flamme, wiederholen. Ein anderer Schritt fährt zur Belegung der Vorderfläche. Die Induction innerhalb des Schellacks, zwischen diesen. Belegen und wiukelrecht gegen sie, ist genau von gleicher Art, wie wenn diese Belege fehlen. Ist $S$ oder der inducirte Körper ein solcher, dafs er der binteren Belegung nicht in Distanz eine Ladung mittheilen kann, ist er z. B. eine unisolirte metallene Kugel oder Platte, damn ist jede dieser Belegungen während der Zeit im polaren Zustand ähnlich dem durch $n p$ in der ersten Figur dieses Briefes abgebildeten, d. h. ihre Vorderflächen haben, wenn der inducirende Körper $P$ positiv ist, eine negative, und ibre Hinterfächen eine positive Ladung und zwar so lange als die Induction dauert.

Ich glaube, Sie zweifeln an dem Daseyn einer specifischen inductiven Capacität. Sie erhalten die Effecte, welche ich auf dieselbe beziehe, scheinen sie aber zu erklären durch einen Act von Leitung in dem Schellack, ähnlich dem in dazwischen gestellten Metallplatten; in der That durch denselben Act, wie Sie ihn voraussetzen, kommt der angenommene negative Zustand der Vorderfläche der Schellackplatte zu Stande. Nun, wenn einer der Inductionseffecte von solcher Leitung herrührte, mülste diese letztere Eigenschaft bei sebr vielen und maunigfaltigen Formen des Experiments auftreten, besonders wenn $Z$ eit in Betracht genommen wird. Ich stellte die Schwefelplatte vor $P$ auf, brachte die Flamme vor die Hinterfläche, entfernte die Platte, stellte die Flamme vor die Vorderfläche, und lud so die Platte negativ und positiv auf den beiden Seiten, wie zuvor beschrieben, in weniger als vier Sekunden und zu bedeutendem Grade. Diese so schìell erlangte Ladung behielt der Schwefel anscheinend ungeschwächt mehre Minuten lang, und woch nach Verlauf von mehren Stunden war er stark geladeu. Wie könnte nuu eine Leitung in- 
nerbalb der Masse des Schwefels (von der Natur der in Metallen vorkommenden ) das Erscheinen der beiden Elektricitäten an seinen Oberflächen in einem Paar Augenblicken veranlafst haben, und zwar von doppelt so grofsem Betrage, als entwickelt seyu würden, wenn Luft dagewesen wäre, - eine Leitung, die dennoch nicht im Stande war, ihre Wiedervereinigung ( return) in einer viele hundert Mal längreren Zeit zu bewerkstelligen? Wir haben Grund zu glauben, dafs die Induction beinah instantan geschieht; denn nehmen wir eine auf dem mittleren 'Theil ihrer beiden Flächen belegte Schwefelplatte und stellen vor ihr in $P$ cine grofse metallene Kugel oder Platte auf, so sind drei successive Contacte, einer zur Ladung von $P$, ein augenblicklicher der Belegung auf der Hinterfläche des Schwefels, und einer zur Eutladung von $P$, hinreichend um den vollen Inductionszustand quer durch den Schwefel zu erregen und die erfolgende Ladung zu sichern. Mittelst einer Taste können diese Contacte im funfzigsten Theil einer Sekunde (fftieth part of a second) vollzogen werden, durch eine kleine mechanische Vorrichtung sogar viel schneller. Dennoch ist, so weit ich finden kann, die belegte Schwe felfläche in dieser kurzen Zeit vollständig geladen, wie wenn die Induction eine Minute und länger gedauert bätte. Wie kömen wir nur denken, dafs irgend ein Grad von Leitung im Schwefel, verträglich mit der längeren Isolation, die darauf folgen kann, zu diesem kurzen und vollständigen Act beigetragen haben könne?

Die vorstebenden Resultate scheinen mir von entscheidendem Charakter zu seyn und keine Frage über die Möglichkeit zu gestatten, dafs bei der Induction die Wirkung eingeschobeuer isolireuder Substanzen von gleicher Natur sey wie die eingeschobener leitender Substanzen. Ich würde weiter eingehen in die Darlegung und Erläuterung meiner Ansichten und der Wirklichkeit einer specifischen inductiven Capacität, wenn ich es für nöthig hielte; allein ich würde wenig mehr zu thun haben als das bereits (und schon vor vielen-Jahren) in der elften Reihe meiner 
Experimental-Untersuchungen Gesagte zu wiederholen und somit stehe ich davon ab.

Der Effect, dessen Sie unten auf p. 404 und oben auf p. 405 des Phil. Mag. erwähnen, ist mir ein sehr natürliches Resultat der bohen specifischen inductiven Capacität des Schellacks. An einer Stelle sagen Sie in Bezug auf dasselbe, "that no reason can be assigned why a small piece of shell-lac etc. "; allein ich kann nicht beistimmen, das für ein kleines Stück anzunehmen, was in Wahrheit ein kleiner, nicht abgetrennter Theil eines grofsen Stückes ist, so wie ich nicht sagen kann, dasjenige sey cin kleines Metallstück, was nur ein kleiner Theil einer grofsen Platte ist. Eine gröfsere inductive Capacität stört die Kraftlinien und Kraftvertheilung in einer Weise, die einem gewissen Betrage von Leitungsvermögen gleichwerthig ist. Und dennoch lassen sich die beiden Effecte vollkommen unterscheiden durch solche Versuche und Schlufsfolgen, wie ich eben auf die Untersuchung des Zustandes der Schellackplatte angewandt habe.

Sie sehen, verehrter Herr, dafs unir daran liegt, gerechtfertigt vor lhnen zu stehen; auch würde ich nicht so weit gegangen 'seyn, wenn ich nicht geglaubt hätte, dals Sie meine Meinungen sebr milsverstanden. Sie werden auch einsehen, dafs ich keinen Grund finde, irgend eine meiner Ansichten über statische Induction, wie sie in Reihe XI aufgestellt sind, zu verändern. Ich mufs bekennen, dafs es, da Ihr Aufsatz in Poggendorff's Annalen und in Philosophical Magazine erschienen ist, mir nicht lieb seyn würde, den Fall vor der wissenschaftlichen Welt so stehen zu lassen, wie er steht, da man glauben könnte, ich genebmigte die daselbst gemachten Angaben; und wenn es lbnen nicht unangenehm wäre, würde ich diesen . Brief in das Magazine geben, es sey denn, Sie zögen eine andere Veröffentlichungsweise vor. Mittlerweile werde ich Ihneu denselben zusenden, und da mehrere Monate seit der $\mathbf{P u}$ blikation hieselbst verflossen sind, so hoffe ich werden Sie 
mir bald Nachricht gebeu, ob Sie dagegen etwas einzuwenden haben oder nicht.

M. Faraday.

Berlin 10. December 1855.

Indem ich auf den Brief antworte, mit dem Sie mich beehrt haben, muls ich zuvörderst Ihre gröfste Nachsicht für mein Englisch in Anspruch nehmen. Ich meine nicht die Fehler, die leicht zu verbessern sind, sondern die unpassende Wahl der Worte, die in theoretischen Controversen von Wichtigkeit wird, und die ich zu vermeiden nicht boffen darf.

Ehe ich an die Erörterung lhrer Bemerkungen gehe in Betreff meines Aufsatzes über die Influenz, mag es nicht unangemesseu seyu, ein Wort zu sagen über die alte Theorie der statischen Elektricität. Es scheint mir, dafs eine Theorie eines Zweiges der experimentellen Wissenschaften für gat erachtet und nicht verlassen werden sollte, so lange sie im Stande ist, alle bekannten Thatsachen aus einem einfachen Principe abzuleiten, sey diefs paradox oder nicht, und so lange sie nicht mit sich selbst oder der Theorie eines verwandten Zweiges in Widerspruch kommt. Die alte Theorie des Lichtes ist verlassen worden, nicht weil ihr Princip von der Aussendung von Myriaden von Lichtheilchen, versehen mit der gröfsten Geschwindigkeit und mancheu verwuuderlichen Eigenschaften, böchlich paradox war, sondern weil sie unzureichend erfunden wurde, über die grofse Klasse von Erscheinungen der Diffraction und Polarisation Rechenschaft zu geben. Ich sehe nicht das Gleiche in der alten Theorie der Elektricität. In der That uimmt sie eine Wirkung in die Ferne an, und ich stimme Ihnen durchaus bej, dafs eine solche Wirkung äufserst schwer zu begreifen ist; aber geben wir nicht dieselbe zu in der grofsen Theorie der Gravitation, und nehmen Sie nicht dieselbe Wirkung an in einem ungewöbn- 
lichen Falle von elektrischer Influenz? ") Die Wirkung in die Ferne besteht hier in der Anziehung von Elektricität der einen Art und Abstofsung der andern in jedem Massentheilchen, und ist unbeschränkt, das will sagen, wenn eine elektrische Partikel $E$ auf ein Massentheilchen $\boldsymbol{A}$ wirkt, und wir ein Massentheilchen $B$ irgendwohin setzen, so wird dadurch die Wirkung von $E$ auf $A$ nicht gehindert oder geschwächt, sondern besteht in demselben Betrage wie zuvor. Giebt man diese Voraussetzungen zu, so erklärt die Theorie die Erscheinungen der statischen Elektricität auf die einfachste Weise. Alle diese Erscheinungen sind Beispiele der elektrischen Anordnung auf der Oberfläche von Körpern, und die Anordnung wird abhängig gedacht von dem Gleichgewichte einer Zahl von Kräften, welche die elektrischen Partikel gegenseitig auf einander ausüben. So werden die elektrostatischen Probleme verwandelt in Probleme der reinen Mechanik, und, die Principien dieser Wisssenschaft finden ihre Anwendung. Der Vortheil dieser Metbode ist sehr grofs; sie stellt den Erfolg jedes Versuches dar als die Summe einzelner Wirkungen, welche das Vorstellungsvermögen obne Schwicrigkeit auffafst, und überläfst der Mathematik die Mühe, die einzelnen Wirkungen zu summiren und den Betrag der Summe anzugeben. Wenn diese Summirung oft zul verwickelt ist, um vollständig ausgeführt zu werden, so halte ich das nicht für einen Febler der Theorie, zumal da es in vielen Fällen nicht schwer ist, mit Hülfe allgemeiner Betrachtungen das Endresultat vorauszusehen. Deshalb habe ich vor langer Zeit diese Theorie vertheidigt gegen ihre - allerdings nicht sehr gefährlichen - Gegner, und ich konnte nicht umhin, die Vertheidigung fortzusetzen,

1) Es ist daran zu erinnern, dafs im Englischen "Induction" sowohl für die Erregung momentaner elektrischer Ströme, als für die Erregung dauernder elektrischer Zustände gebrauclı wird, im Deutschen hingegen von mir das Wort nur für die crste Klasse von Erschcinungen beibehalten, die zweite mit "Influenz" oder Vertheilung bezeichnet worden ist. In dem vorstehenden wie in diesem Schreiben ist ausschliefslich von Influenz die Rede.

$\boldsymbol{R}$. 
als ein Gegner in dem Manne erstand, den ich als den gröfsten Naturforscher unseres Zeitalters verchre.

Auf Ihre erste Bemerkung erwidere ich, dafs ich, einen Fall von Influenz in Luft behandelud, Ihre Meinung über diese Influenz angeführt und absichtlich vermieden habe, Ihre Meinung über einen Fall zu erwäbnen, der nicht vorkam. Denn hätte ich sie erwähnt, so wäre ich gezwungen gewesen hinzuzufügen, daf's Sie nur eine beschränkte Wirkung in die Ferne zugeben [ 1 ], und auseinander zu setzen, dafs diese Annahme für den vorliegenden Fall ganz dieselben Folgerungen zulälst, als wenn Sie jene Wirkung gänzlich leugneten.

In Bezug auf den in der zweiten, Bemerkung gemachten Vorwurf, dafs ich Ihre Meinung über die Wirkung leitender Zwischenplatten bei der Influenz falsch dargestellt habe, mufs ich um so mehr bedacht seyn, mich aufser Schuld zu setzen (disculpate), als diese Rechtfertigung, wenn ich nicht irre, die eigentliche. Wurzel aller Verschicdenheiten berührt, die zwischen Ibrer und der alten Theorie bestehen. Ich habe gesagt, es folge aus Ihren Versuchen, dafs die Einführung einer leitenden Platte zwischen einen influencirenden und einen influencirten Körper die Wirkung des ersten auf den zweiten vermindern würde, weil diese Wirkung, Ihrer Meinung zufolge, jetzt in gekrümmten Linien statt in geraden durch die Luft gehen würde. In diesen Versuchen, die ich angezogen habe, wurde ein geriebener Schellackcylinder und, in Berülirung mit ihm, eine abgeleitete Metallscheibe angewendet, und es wird ein thatsüchlicher Beweis gegeben (exper. resear. 1221) "dafs die Influenz des Schellacks nicht durch oder quer durch das Metall wirkt. " Dieser thatsächliche Beweis besteht in der Beobachtung, dafs eine Prüfungskugel durch Influenz keine oder nur cine geringe Ladung erbält, wenn sie an den Mittelpunkt der oberen Fläche der Scheibe angelegt wird, wo die Kugel dem infuencirenden Körper am nächsten steht, und eine gerade Linie zwischen beiden nur durch das Metall gezogen werden kann; und ferner in der

[1] Siehe Anmerking 1 am Schlufs. 
Beobachtung, dafs die Prüfungskuggel in der Iuft in einiger Höhe über dem Mittelpunkte eine starke Ladung erhält. Hieraus schliefsen Sie: "dafs die Influenz [2] nicht durch das Metall lindurch geschieht, sondern durch die umgebende Luft in krummen Liniet." Ich hielt mich zu der Annahme berechtigt, dafs Sie aus dem gleichen thatsächlichen Beweise den gleichen Schlufs zichen würden in Versuchen von veränderter Form, und ich glaubte diefs um so mehr, als ich keinen andern Weg sah, die Thatsacbe aus Ihrer Theorie abzuleiten. Wenn der geriebene Schellackcylinder durch eine elektrisirte Metallkugel ersetzt, und eine hinlänglich breite Metallplatte in einiger Entfernung über der Kugel befestigt wird, so erhält die Prüfungskugel nur eine schwache Ladung von dem Mittelpunkte der oberen (von der elektrischen Kugel abgewandten ) Fläche der Platte, und eine zunehmende Ladung, wenn sie darüber erhoben wird. Ist die Platte isolirt, so darf die Prüfungskugel nicht an die Platte angelegt werden, aber man betnerkt die Zunahme der Ladung der Kugel mit ihrer Erhebung über den Mittelpunkt, und die stärkste Ladung in einer bestimmten Höhe. Hieraus schlofs ich, dafs Sie die Wirkung einer metallischen Zwischenplatte zwischen einem influencirenden und einem influencirten Körper ansehen würden als eine Schirmung des zweiten vor der Influenz in geraden Linien des ersten [3], und ich wurde in meiner Annahme bestärkt durch \$. 1681 [4]. wo Sie sagen: "dafs die elektrische Kraft beschränkt und ausschliefsend ist."

Sie werden sicher diese Annahme nicht für eine willkürliche erklären, wenn Sie finden, dafs die Anbänger Ihrer Ansichten über die Influenz ganz dieselbe gemacht haben. Melloni glaubte seine Elektroskope vor der Influenz eines Conductors zu schirmen, indem er. eine Metallplatte zwischen beide setzte, und $D$ e la $R$ ive erzählt in demselben Sinne die Versuche mit Ihrem Differential-Inductometer in seinem Traité d'électricité Vol. I, p. 131 (es giebt davon eine englische Ausgabe, die ich nicht gesehen habe).

[2] [3] [4] Anmerkungen 2, 3 und 4 am Schlufs. 
Er sagt: Si on interpose une lame métallique soit isolée, soit, mieux encore, communiquant avec le sol, entre $A$ (dic positiv elektrische influencirende Platte) et $B$ (die influencirte, vorher berührte Platte) aussitôt $B$ donne des signes d'électricité negative très forte, qui proviennent de ce que l'induction cessant d'agir sur elle etc. Ainsi, mettre un disque métallique entre $A$ et $B$, cela revient à remplacer $B$ par un autre disque plus rapproché de $A$ qu'il ne l'était, et par conséquent à le soustraire à l'induction de $A$. Von der metallischen Zwischenplatte, sie sey isolirt oder nicht, wird hier gesagt, dafs sie einen Körper der Influenz entzogen habe, sie wird als ein Schirm betrachtet, der die elektrische Influenz auffängt, wie ein opaker Körpęr das Licht auffängt. Ich bin sehr erfreut, dafs Sie diese Meinung nicht theilen, aber ich mufs gestehen, dafs ich nicht einsehe, wie auf die in Ihrem Briefe dargelegte Weise die Resultate-abzuleiten sind, dic ich mit leitenden Zwischenplatten erhalten habe. Es mag $\boldsymbol{P}$ die ursprünglich elek-
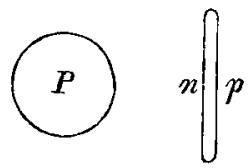

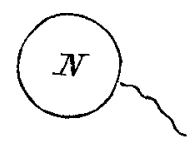
trisirte Kugel vorstellen, $N$ die abgeleitete influencirte Kugel, $\boldsymbol{n} p$ dic (in der Verkürzung gesehene ) isolirte Metallscheibe, die so gestellt ist, dafs die Centrallinie der Kugeln normal durch den Mittelpunkt der Scheibe geht. Ihrer Ansicht zufolge sind die Flächen $n$ und $p$ stärker negativ und positiv, als da die Scheibe aus atmosphärischer Luft bestand (d. i. die Metallscheibe fehlte), und die auf $N$ ausgeübte Influenz sollte kräftiger seyn als vorher. Diefs ist aber in der That nicht immer der Fall; die Influenz auf $N$ erscheint gestärkt oder geschwächt, je nachdem die metallische Zwischenplatte schmal oder breit, dick oder dünn ist. Ich bin nicht im Stande, durch Ihr Räsonnement zu finden, welcher Unterschied eintreten mufs, wenn die Scheibe $n p$ bei gleichem Durchmesser eine Dicke von 0,25 oder von 0,04 eines Zolles besitzt. Es, scheint mir, dafs in beiden Fällen der elektrische Zustand der 
Flïchen $n$ und $p$ gröfser seyn sollte, als bei der Luftscheibe. Dennoch erscheint mit der dicken Metallscheibe die Influenz auf $N$ gröfser, init der dünnen kleiner als in dem Falle, wo keine Scheibe gegenwärtig ist (p. 408 meines Aufsatzes) [5]. Wenn die dicke Scheibe angewendet wird und daher die Influenz auf $N$ verstärkt erscheint, und wir berühren dịe Scheibe einen Augenblick und isoliren sie wieder, so ist die Influenz auf $N$ vermindert. Wollen wir sagen, dafs der gesteigerte Zustand von $p$ durch die momentane Berührung aufgehoben worden, so sollte man erwarten, dafs der Zustand vollständig wieder hergestellt werde bei der folgenden Isolirung der Scheibe [6]. Sey dem, wie ihm șey, ich habe nicht bemerkt, dafs Ihre Theorie die Unabhängigkeit der Wirkungen zugiebt, welche ein influencirender und ein influencirter Körper auf einen dritten Körper ausüben, und diefs ist, wie ich glaube, der wesentliche Punkt, in welchem die beiden Theorien gänzlich von einander abweichen [7]. Die alte Theorie giebt in denkbar einfachster Weise Rechenschaft von allen hieher gehörigen Fällen. Sie nimıt an, dafs die drei elektrischen Schichten: auf der Oberfläche der Kugel $P$, auf der Fläche $n$ und der Fläche $p$ unabhängig von einander influencirend auf die Kugel $N$ wirken. Bezeichnen wir mit $f(P)$ die Wirkung der Influenz der Kugel $P$ auf die Kugel $N$, mit $-f(n)$ die der Fläche $n$ (das Zeichen deutet an, dafs der Effect dem von $P$ erzeugten entgegengesetzt ist), und mit $f(p)$ die Wirkung der Influenz der Fläche $p$, so behauptet die Theorie, dafs in allen Fällen die Gesammtwirkung auf $N$ abhängt von dem Betrage der Summe $f(P)-f(n)+f(p)$ und überläfst der Berechnung zu sagen, ob diese Summe gröfser oder kleiner als $f(P)$ ist. In dem einzigen Fallé, wenn $f(p)$ verschwindet, das beifst, wenn die metallische Zwischenplatte berührt worden oder nicht isolirt ist, kann ohne Berechnung gesagt werden, dafs die Summe der influencirenden Wirkungen von Kugel und Platte kleiner ist als die Wirkung der Kugel allein.

Nachdem ich durch den Versuch gezeigt hatte, dafs mit

Poggendorfl's Annal. Bd. XCVII.

28 
leitenden $\mathrm{Z}$ wischenplatten die Influenz sowohl verstärkt als geschwächt, und mit nicht-leitenden sowohl geschwächt als verstärkt werden kanu, wagte ich die Meinung, dafs die Wirkung von Platten irgend welcher Beschaffenheit auf einem und demselben Grunde beruhe, nämlich auf der Anordnung der Elektricitäten auf den Oberflächen der Platten. Ich untersuchte obenhin (wenn nöthig, könnte es sehr genau geschehen) die Anordnung der Elektricitäten auf einer Metallscheibe und schlors, dafs die Elektricitäten in ähnlicher (nicht derselben) Weise auf der Oberfläche einer nicht-leitenden Scheibe angeordnet sind. Ich gebe zu, dafs dieser Schlufs nicht einwurfsfrei ist, aber ich behaupte, dafs er nothwendigerweise zuerst gemacht werden mufste, und dafs er nicht verworfen werden darf, wenn er nicht als falsch aufgezeigt worden ist.

Die cinfache Thatsache, dafs ein nicht-leitender Körper von einem elektrisirten angezogen wird, zeigt deutlich, dafs ein nicht-leitender Stoff, ebenso gut wie ein leitender, durch die Influenz augenblicklich mit beiden Elektricitäten versehen wird. Als einen mehr directen Beweis der Influenz auf Isolatoren, ohne wesentliche Verbindung mit dem Gegenstaude meiner Abhandlung, habe ich einen Versurh beschrieben, der sich mir darbot und den ich nirgends gefunden hatte. Auf diesen zusătzlichen Versuch bezielst sich Ihre dritte Bemerkung, zu der ich jetzt komme. Eine Schellackscheibe wird schnell von oben nach unten bewegt zwischen einer Flamme und der Kugel des Conductors einer Elektrisirmaschine (nicht to and fro; ich habe diesen Uebersetzungsfehler dem Prof. Ty ndall 19. Juni brieflich angezeigt). Man findet die Vorderfläche der Scheibe stark negativ elektrisch. Sie stimmen mit mir überein, dafs obne die Flamme beide Flächen der Scheibe augenblicklich mit negativer und positiver Elektricität versehen worden sind, aber Sie weichen von mir ab hinsichtlich der Erklärung des Versuches, in Betreff, wie Sie sagen, der Art und Weise, in welcher die Scheibe elektrisirt worden ist, und in Betreff der Rolle, welche die Flamme bei dem End- 
resultate gespielt hat. Was den ersten Punkt angeht, so mufs ein Mifsverständnifs eingetreten seyn, da ich nirgends meine Ansicht über die Weise geäufsert habe, in welcher leitende und nicht-leitende Körper durch die Influenz erregt werden. Ich fürchte, dafs die Ursache des Mifsverständnisses im Worte "distribution " liegt, womit das deutsche Wort "Anordnung " übersetzt worden ist, das die Bedeutung von "arrangement " hat. Wenn gesagt ist ( $p .412$ ) there is no essential difference between the action of conducting and nonconducting bodies, but inasmuch as the distribution of electricity upon them etc. und ferner: in conducting bodies the distribution of electricity etc. - so ist meine Meinung diese: Man giebt zu, dafs jede Zwischenplatte, sie bestehe aus leitendem oder nicht-leitendem Stoffe, durch die Influenz augenblicklich mit beiden Elektricitäten versehen wird, die sich in gewisser Weise auf beiden Platten anordnen. Bei ciner leitenden Platte vermag ich in jedem Falle durch Untersuchung die Anordnung der Elektricitäten anzugeben, und danach die Wirkung dieser Platte auf einen ihr nahe stehenden Körper zu erklären, der von einem elektrisirten Körper influencirt wird. Bei einer nicht-leitenden Platte kann ich zwar die Anordnung der Elektricitäten nicht untersuchen, aber mit einer angenommenen Anordnung kann ich gleichfalls die Wirkung dieser Platte erklären, und muĺs deshalb leugnen, dafs zwischen der Wirkung leitender und nicht-leitender Platten bei der Influenz ein wesentlicher Unterschied vorbanden sey [8]. Dafs die Art und Weise, in welcher die Influenz auf einer leitenden und einer vicht-leitenden Platte zu Stande kommt, in jeder Hinsicht dieselbe sey, habe ich weder gesagt, noch gemeint.

Was den Erfolg des Versuches mit der Flamme betrifft, den ich in meinem Aufsatze angegeben habe, so ist er weder unsicher, noch zweideutig. Die Schellackscheibe wurde einmal von oben nach unten bewegt zwischen dem positiv elektrischen Conductor der Maschine und einer Spiritusflamme; ihre Vorderfläche wurde mit gleitender Berüh- 
rung [9] an den Knopf eines Goldblattelektroskops angelegt; die Scheibe wurde entfernt und die Elektricität des Instruments untersucht. Ueberall wurde negative Elektricität gefunden, stärkere oder schwächere; die stärkste, wenn der Mittelpunkt der Scheibe den Knopf berührt hatte und sorgfältig darauf gebaucht worden war, wovon der Grund einleuchtend ist ${ }^{2}$ ). Ich habe der Flamme die wesentliche Rolle zugetheilt, die positive Elektricität der Hinterfläche zu zerstören, Sie haben die Thatsache beobachtet, dafs die Hinterfläche negativ elektrisch ist, und bieraus einige Folgerungen über die Influenzirung der Platte gezogen, die ich nicht zugeben kann. Bie Thatsache, dafs die Hinterfläche negativ elektrisch ist, scheint mir eine sehr verwickelte zu seyn, und vou einer der beiden folgenden Ursachen herzurühren, vielleicht von beiden. Erstens: Die Flamme wird durch den ursprünglich elektrisirten Körper durch Influenz elektrisirt und theilt ihre negative Elektricität der Hinterfläche mit. Zweitens: Die negativ elektrische Vorderfläche wirkt durch Influenz auf die Hinterfläche. In Bezug auf die erste Annahme habe ich aus Versuchen über die elektrischen Eigenschaften brennender Körper (Pogg. Ann. Bd. 61, S. 545) geschlossen, dafs eine durch Influenz elektrisirte Flamme auf einen ihr nabestehenden Körper mit derjenigen Elektricität wirkt, die der des influencirenden Körpers ungleichnamig ist. Zur zweiten Annahme babe ich einen, wic er mir erscheint, entscheidenden Versuch angestellt und beschrieben im ersten Bande §. 300 meines Buches über Elektricität. Eine Schellackscheibe wurde an eivem Handgriffe frei in der Luft ge-

1) Ich hielt es (mit Unrecht, wie die Anmerkung [9] lehrt) für überGiussig zu bemerken, dals bei den hier beobachteten Divergenzen des Elektroskops die geringe Divergenz nicht in Betracht kommen konnte, welche durch die Reibung des Schellacks gegen den Knopf hervorgebracht wurde. Ueberdiefs fand bei den Versuchen, welche, wie oben bemerkt ist, die stärkste Elektricität lieferten, eine solche Reibung gar nicht statt. Nur der Mittelpnnkt der Schellackscheibe kam mit dem Knopfe des Elektroskops in Berührung und wurde (am bequemsten durch ein Glasrohr) leiclut angehaucht. 
halten und auf einer Fläche (es sey die obere) mit Pelzwerk gerieben. Obgleich nicht $\mathrm{zu} \mathrm{zweifeln}$ ist, dafs die obere Fläche negativ elektrisch war, so wurde auch die untere Fläche negativ gefunden. Hatte dagegen die Schellackscheibe während des Reibens auf einer gut abgeleiteten Metallscheibe gelegen, und war danach die negative Elektricität der oberen Fläche zerstört worden durch Anwendung einer Flamme (oder Berührung mit einer Metallplatte, wie sich sogleich zeigen wird), so wurde die untere Fläche positiv elektrisch gefunden. Nach Zerstörung dieser positiven Elektricität war die obere Fläche wieder negativ, und so fortfahrend, konnte abwechselnd die eine Fläche positiv, die andere negativ gemacht werden. Dieser Versuch gab mir ein leichtes Mittel an, einen Elektrophor mit positiv-elektrischem Kuchen zu erhalten. Dazu wurde der Kuchen in seine gut abgeleitete Metallform gelegt, stark mit Pelzwerk gerieben und in der Form umgekebrt, so dafs die nicht geriebene Fläche oben lag. Wurde dieser Kuchen mit seinem Schilde (einer Metallscheibe) bedeckt, so hatte ich einen Elektrophor, der negative. Elektricităt lieferte, statt dafs der gewöhnliche Elektrophor positive Elektricität liefert.

In Betreff Ihrer vierten und letzten Bemerkung gebe ich durchaus $z u$, dafs es nicht correct ist, einen kleinen Theil eines grofsen Schellackstïckes einem kleinen Schellackstücke gleich zu setzen, was ich p. 405 meines Aufsatzes gethan habe. Aber ich glaube, die Ungenauigkeit unschädlich gemacht $z u$ haben, indem ich dabei auf das Ende meines Aufsatzes hinwies, wo ich erklärt habe, wefshalb eine nicht-leitende Platte bei theilweiser Einführung zwischen einen influencirenden und einen influencirten Körper die Influenz scheinbar schwächt, und sie bei vollständiger $\mathbf{Z w i s c h e n s e t z u n g ~ v e r s t a ̈ r k t . ~ I c h ~ b a l t e ~ n o c h ~ i m m e r ~}$ diese entgegengesetzte Wirkung einer und derselben Platte zusammen mit der Thatsache, dafs die Stellung der Platte zur Seite des influencirenden Körpers die Influenz verstärkt (p. 411 am Ende), für äufserst schwer durch Ihre Theorie der Influenz zu erklären. 
Ich babe wenig Hoffnung, Sie zu überreden, Ihre Ansichten über dic Wirkung der Isolatoren bei der Influenz zu ändern, und ich gestehe, wenn ich es vermöchte, ich würde es kaum wünschen. Der grofse Physiker arbeitet am besten mit Hülfe seiner eigenen Vorstellungen, seiner selbstverfertigten Werkzeuge, deren Unvollkommeuheiten er durch geschickte Anwendung zu vermeiden weifs. Aber diese Werkzeuge, so wirksam in seiner Hand, werden in fremder nicht nur nutzlos, sondern sehr gefährlich, und Sie wissen, welches Unheil zum Beispiel die Vorstellung der elektrischen Schirmung vor Kurzem angerichtet hat in der Hand des seitdem verstorbenen italienischen Physikers. Aus diesem Grunde werden Sie mich nicht tadeln, wenn ich der Veröffentlichung Ihrer Bemerkungen die meiner Entgegnung folgen lassen werde. Gegen Form und Ort, die Sie für diese Veröffentlichung wählen, kann ich keinerley Einwendung machew, und ich weifs, dafs unmittelbar nach dem Erscheinen Ihres Briefés Prof. Poggendorff eine Uebersetzung desselben in seinen Annalen geben wird.

P. Riefs.

Anmerkungen des Hra. Faraday.

1. Meine Ansicht setzt keine Gränze für die Wirkung, welche nicht mit der des Lichtes parallelisirt wird; wo Materie ist, wird diese mit eingeschlossen in die Wirkung, wo sie nicht ist, wird die Wirkung als obne sie geschehend betrachtet.

2. An der abgewandten Seite, indem das Metall immer unisolirt ist.

3. Wenn sie unisolirt ist, ja; isolirt, nein; in Rücksicht auf das Endresultat aller (inducirenden und leitenden) Wirkungen des inducirenden Körpers.

4. "(1681). A striking character of the electric power is that it is limited and exclusive, and that the two forces being always present, are exactly equal in amount. The 
forces are related in one of two ways: either as in the natural normal condition of an uncharged insulated conductor, or as in the charged state, the latter being a case of induction."

5. Die Induction von $P$ geschieht nach meincr Ansicht nicht ausschliefslich auf $N$, sondern auf alle umgebenden Körper, selbst auf die Wände des Zimmers. Wenn die isolirte Metallscheibe $n p$ an Gröfse (size) verändert wird, verändert sich auch die Vertheilung der Induction mit ihr. Eine kleine Scheibe schwächt, wegen Dicke ihrer leitenden Substanz, den elektrischen Widerstand zwischen $P$ und $N$, und vergröfsert also die Induction auf die letztere. Eine gröfsere Scheibe von, derselben oder gar gröfseren Dicke kann durch eine Rückvertheilung der Kräfte die Induction auf $N$ schwächen; es findet nun eine stärkere Induction auf die umgebenden Körper statt, weil ibre Peripherie sich gegen diese mehr ausdehnt.

6. Ich erwarte keine Wiederherstellung des früheren Zustands der Scheibe, und glaube zu wissen, dafs sie nicht erfolgt. Eine momentane unisolirte Berührung bringt augenblicklich einen neuen Zustand der Induction und der Scheibe hervor, welcher endlich ( $/ \mathrm{inal}$ ) ist und nach Aufhebung des unisolirten Contacts verbleibt. Die einzige Störung dieses Zustandes ist die, welche herrührt von der Gegenwart des unisolirenden Drahts, der, während er da ist, Theil nimmt an der Induction auf ihn selbst, (an to itself) - und die, welche die durch Feuebtigkeit und Staub der Luft, sowie durch unvollkommene Isolation veranlafste allmähliche Entladung bewirkt.

7. Meiner Ansicht nach fragt es sich: Ist der Effect der Schellackplatte $n p$ abhängig oder unabhängig von der inneren Leitung zwischen ihren Theilchen? Ist letzteres der Fall, wovon hängt er ab, aufser von der Polarität der Theilchen, welche ich als die Ursache ansehe? Oder nochmals, wie können Leitung und Isolation, betrachtet als beitragende (contingent) Ursachen, als ihr Resultat dieselbe Kraftvertheilung geben? 
8. Gesetzt, ein flüssiges isolirendes Medium sey statt der Luft $z$ wischen $P$ und $N$ vorhanden, und die solide eingeschobene Platte $n p$ habe gleiches Isolationsvermögen und gleiche inductive Capacität wie das Medium, z. B. Schellack in Camphin oder starrer Schwefel in geschmolzenem Schwefel, - dürfen wir erwarten, dafs die beiden Elektricitäten nur an den Oberflächen der soliden Platte erscheinen, und nicht, wie ich voraussetze, in jedem möglichen Querschnitt entweder des Fluidums oder des Solidums, parallel den Oberflächen der Platte oder vielmehr winkelrecht auf den Inductionslinien, gemacht durch Ebenen, welche, wie man annehmen kann, zwischen den Theilchen hindurchgehen, und die Trennung derselben von einander bezeichnen? Würde nicht die erste Voraussetzung seyn, den isolirenden Solidis eine Kraft zuzuschreiben, welche den isolirenden Fluidis abgesprochen wird, und hiefse es nicht so viel als annehmen, dafs das Solidum bei der Induction einen polaren Zustand erlangen könne, welcher jedoch seinen Theilchen abginge? Da die Phänomene der specifischen inductiven Capacität gegenwärtig angenommen sind, ist es sehr wünschenswerth, dafs "die alte Theorie " angebe, wie sie dieselben in unzweifelbaften Fällen, z. B. bein Schwefel oder Schellack, erkläre, und auch erkläre, wie eine nicht-leitende Platte die entgegengesetzten Elektricitäten an ihren beiden Oberflächen entwickelt und verweilend haben kann ohne Leitung oder ohne das, was ich Polarisation genanut habe.

9. Ich gah die eine Bewegung zwischen dem inducirenden Körper und die Flamme, und erhielt genau dieselben Resultate wie die in meinem Briefe beschriebenen. Es ist, obne je die Kugel oder Deckplatte des Elektrometers zu berühren, blofs durch starkes Nähern, ganz leicht zu ermitteln, welche Fläche der Platte $n p$ geladen sey, und ob positiv oder negativ. Ich balte es für wesentlich nöthig, einen schleifenden Contact zwischen der Schellackplatte und der Metallkugel des Elektrometers zu vermeiden, denn ich finde, dafs, wewn man zwischen der voll- 
kommen ungeladenen Platte und dem Instrument einen solchen Contact vollzieht, Elektricität erregt wird, der Schellack positiv und das Metall negativ wird, so dafs nach Fortziehung des Schellacks das Elektrometer mit negativer Elektricität divergirt. Wird ein geladenes Stück trockner Schellack (durch Reibung mit Metall positiv gemacht und stark genug um bei Annäherung an ein Elektrometer die Goldblättchen um einen Zoll und mehr divergiren zu machen) angewandt, so finde ich es unmöglich diese Ladung zu dem trocknen Instrument durch Reibung an ihrer Metallkappe überzuführen; der Schellack wird nur positiver und hinterläfst das Instrument in negativen Zustand; deshalb zweifle ich an der einfachen Mittheilung der negativen Elektricität von schwach geladenem, trocknen, isolirtem Schellack an trocknes Metall durch reibenden Contact; aber ich erwarte in jedem Fall Erregung (excitement and evolution) von Elektricität, und dafs das Elektrometer negativ, der Schellack positiv werde.

\section{Ueber die Anwendung der mechanischen WVärme- theorie auf die Dampfmaschine; oon R. Clausius.}

1. Da die veränderten Ansichten über das Wesen und das Verhalten der Wärme, welche unter dem Namen der "mechanischen Wärmetheorie " zusammengefafst werden, in der bekannten Thatsache, dafs sich die Wärme zur Hervorbringung von mechanischer Arbeit anwenden läfst, ihre erste Anregung gefunden haben, so durfte man im Voraus erwarten, dafs die so entstandene Theorie auch umgekehrt wieder dazu beitragen müsse, diese Anwendung der Wärme in ein helleres Jicht zu stellen. Besonders mufsten die durch sie gewonnenen allgemeineren Gesichtspunkte es möglich machen, ein sichreres Urtheil über die 